\title{
Current Diagnostic Methods in the Detection of Oral Cancer- An Update
}

\section{Sunitha HM*, Amandeep Sodhi and Vathsala Naik}

Post Graduate Student, Department of Oral Medicine and Radiology, Bangalore

Institute of Dental Sciences and Hospital, Bangalore, India

*Corresponding Author: Sunitha HM, Post Graduate Student, Department of

Oral Medicine and Radiology, Bangalore Institute of Dental Sciences and Hospital,

Bangalore, India.

DOI: $10.31080 /$ ASDS.2020.04.0975
Received: October 15, 2020

Published: November 09, 2020

(C) All rights are reserved by Sunitha HM., et al.

\begin{abstract}
Oral cancer is the $6^{\text {th }}$ most common cancer in the world with significant morbidity and mortality which is frequently associated with local lymph node metastasis. As the prevalence of the disease is increasing and the survival rate has remained low for decades, it is essential to diagnose the disease at an early stage. Early diagnosis of the disease significantly increases the probability of the cure of oral squamous cell carcinoma with minimal impairment and deformity of the associated structures. Various methods in the diagnosis of Oral cancer are visualization methods, vital staining which includes - Toluidine Blue, Lugol's iodine, Methylene blue, laboratory based diagnostics- Biopsy, salivary biomarkers, Light based diagnostic tools, salivary biomarkers, advanced imaging modalities. This article reviews the conventional and latest diagnostic methods which aids in not only early detection but also in accurate treatment planning of oral cancer.
\end{abstract}

Keywords: Oral Cancer; Vital Staining; Light Based Diagnostic Methods; Salivary Biomarkers; Advanced Imaging

\section{Abbreviations}

OSCC: Oral Squamous Cell Carcinoma; PMD: Premalignant Disorders; TB: Toluidine Blue; RS: Raman Spectroscopy; ESS: Elastic Scattering Spectroscopy; DPS: Differential Path-Length Spectroscopy OCT: Optical Coherence Tomography; A/LCI: Angle-Resolved Low Coherence Interferometry; FTIR: Fourier Transform Infrared Spectroscopy; OPG: Orthopantomogram; PNS: Para Nasal Sinus; IOPAR: Intra Oral Peri Apical Radiograph; CT: Computed Tomography; CBCT: Cone Beam Computed Tomography; MRI: Magnetic Resonance Imaging; HR USG: High Resolution Ultrasonography; PET: Positron Emission Tomography; DNA: Deoxyribonucleic Acid; RNA: Ribonucleic acid; MGMT: Methylguanine-DNA Methyltransferase; DAPK: Death Associated Protein Kinase; MMP: Matrix Metalloproteinase; TGF: Transforming Growth Factor; TNF: Tumour
Necrosis Factor; IL: Interleukin; DUSP: Dual Specificity Phosphatase; OAZ: Ornithine Decarboxylase Antizyme; SAT: Spermidine/ Spermine N1-Acetyltransferase; LBDS: The Light Based Detection Systems; NMR: Nuclear Magnetic Resonance; VEL: Visually Enhanced Lesion; 5-ALA: 5-Aminolevulinic Acid; PPIX: Protoporphyrin IX; NAD: Nicotinamide Adenine Dinucleotide; FADH: Flavin Adenine Dinucleotide; STIR: Short Tau Inversion Recovery; TIRM: Turbo Inversion Recovery Magnitude; DWI: Diffusion Weighted Imaging

\section{Introduction}

Oral cancer is the $6^{\text {th }}$ most common cancer in the world and $8^{\text {th }}$ leading cause of death worldwide. $90 \%$ of Oral cancers have their origin in the squamous epithelial cells and hence are called squa- 
mous cell carcinoma [1]. Oral cancer is associated with significant morbidity, which metastasizes to regional lymph nodes of the neck, distant sites like lung, liver and bone causing an eventual mortality [2].

Like all cancers, oral cancer is also a molecular disease with genetic and environmental influences. Mostly it is associated with habitual tobacco chewing, smoking, betel nut chewing, consumption of alcohol, and Human Papilloma Virus [3].

Oral cancer is easily accessible and tumours at this site present with symptoms at a much early stage. Even then unfortunately a large number of patients are seen to report with late stage diseases [4]. Hence the mortality rate still remains at $49 \%$ and the overall disease-free survival rate of 5 years has been just about $50-60 \%$ for many decades $[2,5]$. The only way to reduce the mortality rates and improve the 5-year survival rates, is in early detection and prevention of the disease by various early diagnostic approaches. The gold standard for the diagnosis of Oral cancer is a thorough clinical examination combined with a histopathological investigation [6]. However recent advancements in cancer diagnostics, aids in a more focused and less invasive approaches for early detection, leading to a better monitoring of the therapeutic response [1].

\section{Methods of diagnosis}

The methods in the early detection of oral cancer includes oral cancer screening programs that aids in the identification of asymptomatic patients with suspicious lesions and to employ specific diagnostic tools to identify dysplasia and early oral cancers in asymptomatic patients with an oral abnormality. The benefits and limitations of these methods will be discussed in this review.

\section{Diagnostic aids for oral cancer}

\section{Clinical methods/chairside investigations}

\section{A. Conventional methods:}

I. Visual method

II. Vital staining:

- Toluidine blue

- Lugol's iodine

- Methylene blue.

III. Oral brush biopsy.

\section{B. Advanced techniques:}

- ViziLite/chemiluminiscence/tissue reflectance

- $\quad$ ViziLite plus

- VEL scope/visual autofluorescence

- Autofluorescence imaging

- $\quad$ Fluorescence spectroscopy

- Enhanced dye fluorescence

- $\quad$ Ratio imaging

- $\quad$ Raman spectroscopy (RS)

- $\quad$ Elastic scattering spectroscopy (ESS)

- Trimodal spectroscopy

- Differential path-length spectroscopy

- Optical tomography

- $\quad$ Optical coherence tomography (OCT)

- $\quad$ Fourier transform infrared (FTIR) spectroscopy.

Laboratory investigations

\section{Conventional:}

- $\quad$ Biopsy, FNAC.

\section{Blood and salivary investigations}

- Biochemical markers

- Genetic markers.

\section{Imaging modalities:}

\section{Anatomical:}
a) Conventional Radiology: OPG, PNS, Occlusal, IOPAR
b) CT, CT guided Biopsy
c) $\mathrm{CBCT}$
d) MRI
e) HR- USG, USG-FNAC. 


\section{Functional:}
a) Nuclear Scintigraphy
b) PET/CT.

\section{Vital staining \\ Toluidine blue}

It is a vital, acidophilic metachromatic dye of thiazine group which stains the tissues rich in acidic component like deoxyribonucleic acid and ribonucleic acid. As there is increased DNA synthesis and also random arrangement of tumour cells, there is a rapid penetration of the dye in the premalignant and malignant tissues. Dark blue stain helps to differentiate areas of carcinoma in situ and invasive carcinoma which have more dysplastic and anaplastic cells than the normal tissues. Advantages, it is a simple, non- invasive and cost effective. Disadvantages - alteration in taste, stains remaining in the mouth for sometime and false positive results in cases of hyperkeratosis or sensitivity varies from $78-100 \%$ and specificity from $62 \%[7,8]$.

\section{Lugol's iodine}

Iodine reacts with glycogen in the cytoplasm and the reaction, known as the iodine-starch reaction can be visualized by a colour change. The glycogen content of the tissue is linked to the degree of keratinization. since there is increased keratinization in malignant cells along with the loss of cellular differentiation and enhanced glycolysis which depletes the glycogen in cancer cells, these cells do not promote the iodine-starch reaction [9]. Brown to black stain is noticed in the glycogen rich normal mucosa, whereas no retention of stain is observed in dysplastic cells which turn mustard yellow to saffron in colour $[8,10,11]$. Advantages are simple, non-invasive, inexpensive and highly sensitive. Disadvantages are it causes irritation to the normal mucosa, heart burn, nausea, allergic reactions to iodine [12].

\section{Methylene blue}

Methylene blue is an odourless, dark green colour powder at room temperature which changes to blue colour when mixed with water. The mechanism is similar to Toluidine blue as methylene blue is also an acidophilic dye. The uptake of the dye, dark blue colour indicates malignancy, light uptake of the dye indicates premalignant lesion whereas no uptake of the dye indicates normal tissue. It is a simple, inexpensive, non- invasive procedure with sensitivity $95 \%$ and specificity $70 \%[13]$.

\section{Biopsy}

Biopsy procedure is a surgical procedure for obtaining of a living tissue sample for performing diagnosis. The current gold standard for diagnosis is the histopathologic examination of the suspicious lesion which is obtained by biopsy. There are different types of biopsy like incisional biopsy, excisional biopsy, scalpel biopsy, punch biopsy, B- forceps, Frozen section, fine needle aspiration biopsy and oral brush biopsy [14].

\section{Oral brush biopsy/oral CDx}

Oral CDx is a special technique which with the aid of a computer can analyze the suspected lesion. A specially designed round brush with stiff bristles is placed on the lesion and rotated until it produces a hemorrhagic spot, which indicates the inclusion of the sufficient sample thickness. This tissue is then fixed on a glass slab using a fixative agent which is available in brush biopsy kit. This sample is then sent to CDx Laboratory where it is analyzed with the help of a computer. Advantages are, it is easy to perform, noninvasive, less bleeding and less painful compared to scalpel biopsy. Disadvantages are, it detects only cellular atypia, is time consuming and expensive [15]. Gupta., et al. combined conventional oral brush biopsy with the application of TB to identify suspicious mucosal areas. Sensitivity is $43-71 \%$ and specificity is 81 to $97 \%$ [16].

\section{Saliva-based oral cancer diagnosis}

Saliva is an easily available specimen which contains a large number of proteins and peptides that can be used as a biomarker for the diagnosis of various oral and systemic diseases. Molecular diagnosis of oral cancer can be carried out in three levels i.e., Changes in the cellular DNA, altered mRNA transcripts and Altered protein levels intra-cellularly or extra-cellularly. Hence, saliva biomarker can be a genetic marker, protein marker or a transcriptome marker [17]. 


\section{Genomic markers}

Genomic markers consists of DNA and RNA markers and are driven by the presence of specific genetic variations. DNA shows various tumor-specific features such as somatic mutations in tumor suppressor genes and p53, microsatellite variation, abnormal promoter methylation, mitochondrial DNA mutations, and presence of viral DNA related to the tumour. Promoter hyper methylation of one of the three genes (p16, MGMT, or DAP-K), detection of telomerase activity can be used as an additional marker [18].

\section{Salivary protein markers}

Some of the salivary proteins which are elevated in oral squamous cell carcinoma for example, defensin-1, which is present in the early stage of the cancer. Several protein markers like interleukins $(8,6,1 b)$, matrix metalloproteinase (MMP 2, 9), transforming growth factor (TGF-1), Ki67, cyclic D1, Cyfra 21.1, transferrin, a amylase, tumour necrosis factor (TNF-a) and catalase can be detected in saliva of oral cancer patient [17].

\section{Transcriptome markers}

Salivary transcriptome diagnostics is a novel clinical approach where a large panel of human RNAs are readily detected in the saliva. MicroRNAs and small RNA molecules, 18 - 24 molecules in length, that are known to regulate transcription were discovered in the saliva samples. Li., et al. used the microarray analysis of the salivary transcriptome which showed that seven markers showed a significant increase in the saliva of the OSCC patients. The advantages of salivary biomarkers are it is inexpensive, non-invasive, cost effective and easily accessible media and much safer when compared blood. The disadvantages are Lack of standardization in sample collection, processing and storage and Variability in the levels of salivary biomarkers [17].

\section{Light-based detection systems}

The light based detection systems (LBDS) in the recent years have become more acceptable due to their non- invasive nature in the detection of premalignant lesions and oral squamous cell carcinoma. It uses the optical properties of biological tissues in enhancing oral mucosal examination.
ViziLite is a chemiluminescent based detection device which is designed for the detection of PMD and OSCC. The patient is asked to rinse the mouth with $1 \%$ acetic acid solution for 1 minute for the purpose of drying the oral mucosa and disruption of glycoprotein barrier. Then the bluish white light with a wavelength of 430 - 580 $\mathrm{nm}$ is passed over the oral tissue. Normal tissue absorbs light and appear dark with a blue hue, whereas abnormal or malignant tissue reflect the light and appear "aceto-white" with brighter, sharper and distinct margins. Advantages are it is simple, non-invasive, gives better demarcation between normal and abnormal tissue. Disadvantage is low specificity i.e. $27.8 \%$ and sensitivity is about $77.3 \%$ [20]. For better visibility a modified version of ViziLite is developed which is called ViziLite Plus, which uses chemiluminescence and Toluidine Blue (TB) marking system [20].

\section{Visual autofluorescence (Visually enhanced lesion scope)}

VELscope device is a hand held, non- magnifying device which uses $400-460 \mathrm{~nm}$ wavelength light. The light emitted from the device reaches the oral mucous membrane and excites the endogenously present auto-fluorescent substances called fluorophores. The normal tissue will fluoresce more because of the presence of fluorophores and emit apple green colour, whereas malignant tissue has decreased fluorescent properties and appear dark. Advantages are VELscope machine is portable, easily stored and it is painless quick procedure. It has a sensitivity of $74.1 \%$ and a specificity of $96.3 \%$ [21]. Disadvantage is that the increase of haemoglobin in inflammatory hyperaemia site may enhance the absorption of light leading to fluorescence deficiency, which gives false positive result of VEL scope [22].

\section{Autofluorescence imaging}

In autofluorescence imaging the tissues are illuminated with a light source, in the near UV to the green spectral range. The fluorescence images which are produced by the tissue are recorded in the camera. Digitally captured images are then used to interpret the lesion. The tissue appears green in color in both normal and cancerous cells. The cancerous tissues have low autofluorescence intensities as compared to normal tissue as there is loss of collagen stroma. Thus, the cancerous lesions can be differentiated from 
the normal tissue by a darker shade of green. The advantage of autofluorescence imaging when compared to that of VELscope is that visual autofluorescence is always subjective to the observer's skill where as in case of autofluorescence imaging digital images are captured and they can be compared with the normal algorithm chart to find out high risk or lesional areas [22].

\section{Autofluorescence spectroscopy}

Autofluorescence spectroscopy is based on the absorption of ultraviolet and visible light spectrum by fluorophores present in the tissue molecules that leads to emission of lower energy photons which is detected as fluorescence from the oral mucosa. Optical fibres are introduced into tissues using a hollow needle and then the tissue signals are interpreted by the spectrometers. Betz., et al. compared autofluorescence imaging and spectroscopy of normal and malignant mucosa in $43.3 \%$ of patients it was easier to distinguish tumours from adjacent normal mucosa by observing reduction in green autofluorescence, whereas in Spectral analysis 94\% patients showed contrasts in autofluorescence intensities between tumour and normal tissues. Mayinger conducted an endoscopic autofluorescence spectroscopy on oesophageal cancer and they obtained a sensitivity and specificity of $97 \%$ and $95 \%$ respectively [23].

\section{Enhanced dye fluorescence}

Fluorescence can be enhanced by exogenously applying fluorescent drugs such as 5-aminolevulinic acid (5-ALA), which induces protoporphyrin IX (PPIX) It is an important precursor to biologically essential prosthetic groups like heme, cytochrome c, and chlorophylls). In addition it is possible to exactly target tumour-specific enzymes with fluorescent markers, which will improve both sensitivity and specificity [24].

\section{Ratio imaging}

Ratio imaging compares a photochemical or end metabolic product which could be increased in disease states with another product that is known to be depleted. As mentioned above, 5-ALA enhances PPIX, which fluoresces red after excitation with blue light. The same excitation results in green fluorescence of mol- ecules such as NAD and FADH, which are depleted in malignant tissues with a high metabolic rate. Shin., et al. have reported that the sensitivity of fluorescence imaging is about 60 to $97 \%$ and specificity is 75 to $99 \%$ [24].

\section{Raman spectroscopy (RS)}

Raman spectroscopy (RS) is a vibrational spectroscopy method based on the inelastic scattering of light. When the tissue is irradiated with intense monochromatic light, there is absorption, scattering and reflection of the light. Most of the scattered photons have the same frequency of the incident light while a small proportion (one in ten million) have inelastically scattering properties, i.e. with a frequency different from the incident photons; this phenomenon is termed as Raman effect. A continuouswave laser is employed as the excitation source in Raman spectrometers [24]. Advantages are it can be applied to wide range of samples, minimal preparation of the tissue i.e. no fixation, non-invasive, procedure. Disadvantages are production of week signals, biomedical samples produce strong fluorescence, over lapping of Raman band. Further research needs to be done [25].

\section{Elastic scattering spectroscopy (ESS)}

Elastic Scattering Spectroscopy (ESS) uses objective statistical and analytical methods rather than by subjective interpretation of images. It gives optical geometrical information that is based on white light reflectance. In ESS, the photons hit the tissue which are backscattered without any wavelength changes. The backscattering is influenced by the composition of the interrogated tissue, specifically the relative concentration of scatterers (e.g. nuclei, mitochondria, connective tissue) and absorbers (e.g., haemoglobin). A scattering event carries with it all the characteristics of the cellular components, which are called "scattering centres". Pathological scattering originates from disorganised epithelial orientation and architecture [24].

\section{Differential path-length spectroscopy (DPS)}

Differential path-length spectroscopy is a form of ESS that has fixed photon path length, visitation depth, and absolute measurement of absorbers. DPS uses a fibre-based diffuse reflection spec- 
trometer with a tungsten-halogen lamp as a white light source. The first spectrometer uses a bifurcated fibre for illumination and collection. A second fibre carries diffusely reflected light to a second spectrometer. The spectrum is analyzed mathematically and is translated into a set of parameters which are related to the microvasculature and intracellular morphology. The sensitivity and specificity reported was $69 \%$ and $85 \%$ respectively $[24,26]$.

\section{Optical tomography}

Optical Tomography uses light scattering to either construct an image like an OCT, or to measure the average size of different cell structures, which provides objective information about degree of dysplasia, such as angle-resolved low coherence interferometry (a) LCI) [24].

\section{Optical coherence tomography (OCT)}

OCT is a lightbased diagnostic tool that uses backscattered reflections to create images up to a depth of 1.5 - $2 \mathrm{~mm}$ and conceptually it is very much similar to ultrasound. OCT is a non invasive procedure with realtime imaging which helps in obtaining images that could be compared to histopathological sections and hence this procedure is also known as optical biopsy. The contrast in OCT images is due to the difference in lightabsorbing capacity of tissues, whereas in histopathology specific stains are used to study cellular and subcellular components. therefore, OCT can be used as an adjunct but not analogous to histopathology. Advantages are simple, quick, non-invasive whereas the limitations are in cases of haemorrhage, there is will be strong attenuation of light which results in shadowing of deeper structures, limited depth of penetration, image quality is compromised in oral pre malignancies due to hyperkeratosis. Fomina., et al. conducted a study which had specificity $83 \%$ and $98 \%$ specificity [27].

\section{Nuclear magnetic resonance spectroscopy (NMR)}

NMR uses the magnetic properties of some atomic nuclei to determine the physical and chemical properties of atoms or the molecules in which they are contained. It is based on the phenomenon of nuclear magnetic resonance which can provide detailed information about the structure, dynamics, reaction state and chemical environment of molecules. It allows three dimensional study of at- oms in molecules; the larger the magnet, more sensitive is the device. NMR has been used to identify metabolic signatures of OSCC compared with normal tissues. Studies have shown that the choline/creatine ratio is significantly increased in OSCC than in normal tissue [22]. Advantages are multiple metabolites are acquired in single measurement, no radiation exposure. Limitations are long acquisition time, lack of familiarity to the clinicians [29].

\section{Fourier transform infrared (FTIR)}

Fourier Transform Infrared (FTIR) spectroscopy can be used to detect cancer at an earlier stage. Any variation in the biochemical distribution in the cells during the carcinogenesis could act as an early marker for the cancer detection, before even morphological changes could occur. The Infrared spectroscopy monitors the global chemical composition of the sample. Variations of collagen structure can be demonstrated by FTIR spectroscopy. FTIR spectroscopy along with imaging can be used for the quantitative analysis of tissue, cells and bio-fluids in the clinical practice [30,31].

\section{Imaging modalities}

Conventional radiography: Conventional imaging techniques with x-rays are the most familiar type of imaging for screening of oral cancer. A combination of OPG and intraoral radiography can be used in the in the detection of bone invasion particularly in determining the supero-inferior extent of the lesion in mandibular gingiva region. The characteristic features of malignant lesions on plain radiographs are thinning of cortical lamina, osteolytic defects with both unilocular or multilocular initial osteosclerotic capsule, whereas in later stages the defective bony ridges become sharp and the teeth lose their bony support. A combination of OPG and Scintigraphy has high specificity and sensitivity and hence gives accurate results. These simple techniques also have added advantages like economical, easy availability, familiarity to the clinician, easy and most practical approach in the screening of oral cancers. The drawbacks of plain $\mathrm{X}$ ray is its inability to image soft tissue, false positive due to periodontitis, erosion of the bone can be visualised only after $30-75 \%$ mineral loss and limited visibility of the symphysis region. OPG can be used in assessment of bone involvement with a sensitivity of $75 \%$ and specificity of $100 \%[32,35,40]$. 


\section{Computed tomography (CT)}

Computed tomography is a three dimensional, standard tool used to detect primary tumours and to know their bony infiltration. Special attention should be given to small tumours with a high degree of differentiation as the contrast enhancement can be scarce. However, lesions with a low degree of differentiation can be easily distinguished from the surrounding tissues. Contrast-enhanced CT accurately determines lymph node metastases. The sensitivity of CT in detecting tumors is $41-82 \%$ and specificity $82-100 \%$ [33].

\section{Perfusion CT}

As there is increased blood volume and blood flow in the tumours, it results in neoangiogenesis. It is considered hat perfusion CT is superior to CT as there is better assessment of muscle involvement. The sensitivity of Perfusion CT in detecting local lymph node metastases is $67 \%$, specificity is $53 \%$ [33].

\section{CT- guided biopsy}

Has also gained acceptance because of its safety and high accuracy. However, the drawbacks with the use of CT are presence of amalgam fillings and metallic prosthesis in the oral cavity which can result in artifacts and obscure the anatomy and pathology. A CT scan is not as useful in cases where soft tissue tumours are to be studied. Hence a CT is not recommended in cases of carcinomas of tongue and gingiva [36,37].

\section{Cone beam computed tomography (СВCT)}

CBCT is a relatively new imaging modality that uses a cone shaped X-ray beam to capture a 3D image in one sweep. The advantage of CBCT is smaller and less expensive, lower radiation dose when compared to CT, and allows the patient to be in an upright position for the examination, thereby limiting the effects of gravity on soft tissues. However, CBCT is limited by a poor assessment of soft tissues (sensitivity 89 - 93\%, specificity 60 - 96.5\%) [33,34].

\section{Magnetic resonance imaging (MRI)}

MRI is mainly used for soft tissue evaluation of mainly tongue and floor of the mouth. T1, T2, STIR, TIRM, DWI, perfusion with or without a contrast agent are the sequences used in MRI. It enables the detection of very small lesions, assessment of local spread of the tumour and planning surgery. MRI can determine the involvement of local soft tissues, bone marrow and bones (sensitivity $82 \%$, specificity $66.7 \%$ ] [33].

\section{Ultrasonography (USG)}

Ultrasound is produced by vibration of piezoelectric crystals with the help of a highfrequency electrical pulse, which causes mechanical oscillation producing ultrasound waves. Diagnostic ultrasound utilizes a transducer, which generates a narrow focus beam. And this beam is reflected from the tissue and sent back to the same transducer, which then assembles these echoes into an image that is visualized and recorded. USG has several advantages like it is harmless, radiation free, widely available, easytouse, non-invasive, inexpensive, and unaffected by metal artefacts, it can differentiate between benign and malignant lymph nodes, can be repeated if necessary. USG with color-Doppler is used to assess the involvement of lymph nodes. Ultrasonography is mainly used in the evaluation of superficial lesions, lymph nodes and also to guide needle aspiration biopsies (sensitivity 79\%, specificity 69\% for needle aspiration biopsies) [33].

\section{Bone scintigraphy}

Bone imaging using radionuclides is a non-invasive technique which demonstrates the osteoblastic lesion of the skeletal system. This is based on the uptake of a radiotracer which happens when the bone is being formed or repaired. It is very helpful in tumors of head and neck which mostly invade the adjacent bones. A bone loss upto 5 percent can also be accurately detected with a bone scan. It is economical and has a high sensitivity rate. The drawback of this technique is that it lacks specificity. The metabolic changes may occur in several benign dental conditions like healing extraction wounds, dental abscesses, periodontal abscesses, pericoronitis and osteomyelitis and could produce false positive bone scintigrams $[35,36]$.

\section{Positron emission tomography (PET)}

PET with 18F-fluorodeoxyglucose evaluates metabolic activity of the tissue, as there is increased metabolic activity in the tumour cells. It is used when planning adjuvant treatment and also for prediction of survival without recurrence. It can be used for the detection of metastatic lymph nodes (sensitivity $83 \%$, specificity $88 \%$ ) [33]. 


\section{Conclusion}

Oral cancer poses an important threat to the overall survival of an individual. The ability subsequently help in better disease management. The ultimate goal is to lower the morbidity and mortality and hence help in improving patients' quality of life. Early diagnostic techniques are becoming more easy to use, accurate and less invasive hence they are beneficial not only in differentiating normal mucosa from dysplastic or malicious lesions, but they also help in monitoring treatment and keep a check on recurrences. Integration of the adjuncts and diagnostic aids discussed here can help uncover hidden lesions before they even progress to an advanced stage. In conclusion, all diagnostic methods have their advantages and limitations. Hence, it is the responsibility of the practitioner to combine them in order to attain the highest possible efficacy and sensitivity, as this can significantly improve the treatment outcome.

\section{Acknowledgements}

I would like to acknowledge Dr. Anisha Yaji, MDS, Oral Medicine and Radiology for her constant support and guidance.

\section{Conflict of Interest}

No conflict of interest.

\section{Bibliography}

1. Cristaldi Marta., et al. "Salivary biomarkers for Oral Squamous Cell Carcinoma diagnosis and follow-up: current status and perspectives". Frontiers in Physiology 10 (2019): 1476.

2. Limeback Hardy. "Comprehensive preventive dentistry". John Wiley and Sons (2012).

3. Grafton-Clarke., et al. "Diagnosis and referral delays in primary care for oral squamous cell cancer: a systematic review". British Journal of General Practice 69.679 (2019): e112-e126.

4. Agar NJM and RS Patel. "Early detection, causes and screening of oral cancer". JSM Dental Surgery 2.3 (2014): 1039.

5. Byrd David R., et al. "AJCC cancer staging manual". Edition. Stephen B. Edge. New York: Springer 649 (2010).
6. Ebrahimi Ardalan., et al. "Depth of invasion alone as an indication for postoperative radiotherapy in small oral squamous cell carcinomas: An International Collaborative Study". Head and Neck 41.6 (2019): 1935-1942.

7. Singla S., et al. "Conventional and Advanced Diagnostic Tools in Oral Cancer with Emphasis on Role of Dentist and Early Detection". The Journal of Postgraduate Medicine, Education and Research 51.3 (2017): 128-133.

8. Warnakulasuriya KAAS and Newell W Johnson. "Sensitivity and specificity of OraScan ${ }^{\circledR}$ toluidine blue mouthrinse in the detection of oral cancer and precancer". Journal of Oral Pathology and Medicine 25.3 (1996): 97-103.

9. M Petruzzi., et al. "Use of Lugol's iodine in oral cancer diagnosis: An overview". Oral Oncology 46 (2010): 811-813.

10. Hasan SHAMIMUL and S Elongovan. "Conventional and advances diagnostic aids in oral cancer screening-The journey so far". International Journal of Pharmacy and Pharmaceutical Sciences 7 (2014): 29-33.

11. Nagaraju Kamarthi., et al. "Diagnostic efficiency of toluidine blue with Lugol's iodine in oral premalignant and malignant lesions". Indian Journal of Dental Research 21.2 (2010): 218.

12. Masthan KMK., et al. "Advanced diagnostic aids in oral cancer". Asian Pacific Journal of Cancer Prevention 13.8 (2012): 35733576.

13. Reddy., et al. "Oral screening techniques". Journal of Orofacial Sciences 6.1 (2014).

14. Abraham Lejoy., et al. "Methylene blue as a diagnostic aid in the early detection of potentially malignant and malignant lesions of oral mucosa". Ethiopian Journal of Health Sciences 26.3 (2016): 201-208.

15. Poh Catherine F., et al. "Biopsy and histopathologic diagnosis of oral premalignant and malignant lesions". Journal of the Canadian Dental Association 74.3 (2008): 238-288. 
16. Bhosale Satish and Tarun Vyas. "Application of Oral CDx Brush Biopsy in Oral Cancer Detection". (2019).

17. Reddy Sridhar G., et al. "The sensitivity and specificity of computerized brush biopsy and scalpel biopsy in diagnosing oral premalignant lesions: A comparative study". Journal of Oral and Maxillofacial Pathology: JOMFP 16.3 (2012): 349.

18. Radhika T., et al. "Salivary biomarkers in oral squamous cell carcinoma-An insight". Journal of Oral Biology and Craniofacial Research 6 (2016): S51-S54.

19. Wong David T. "Salivary diagnostics powered by nanotechnologies, proteomics and genomics". The Journal of the American Dental Association 137.3 (2006): 313-321.

20. Saxena Shikha., et al. "A review of salivary biomarker: a tool for early oral cancer diagnosis". Advanced Biomedical Research 6 (2017): 90.

21. Sambandham Thirugnana., et al. "The application of vizilite in oral cancer". Journal of Clinical and Diagnostic Research: JCDR 7.1 (2013): 185.

22. Zhou Jiaying., et al. "Non-Invasive Approaches for Oral Potential Malignant Disorders Surveillance: A Review". Advances in Bioscience and Biotechnology 11.5 (2020): 188-205.

23. Sawan Dania and Ammar Mashlah. "Evaluation of premalignant and malignant lesions by fluorescent light (VELscope)". Journal of International Society of Preventive and Community Dentistry 5.3 (2015): 248.

24. Mascitti Marco., et al. "An overview on current non-invasive diagnostic devices in oral oncology". Frontiers in Physiology 9 (2018): 1510.

25. Chaturvedi., et al. "Noninvasive early diagnosis of oral lesions". Journal of Cancer Research and Therapeutics 6.4 (2010).

26. Balasubramaniam., et al. "Autofluorescence based diagnostic techniques". Journal of Pharmacy and Bioallied Sciences 7.2 (2015): 374-377.
27. Alqhtani Nasser., et al. "Raman spectroscopy and oral cancer". Head and Neck Oncology 1.1 (2009): 1-1.

28. Sahu and Krishna. "Raman spectroscopic applications in oral cancer". Journal of Cancer Research and Therapeutics 13.6 (2017).

29. Reddy and Praveen. "Optical coherence tomography in the diagnosis of oral cancer". Journal of Cancer Research and Therapeutics 13.6 (2017).

30. De Coro Michael and Petra Wilder-Smith. "Potential of optical coherence tomography for early diagnosis of oral malignancies". Expert Review of Anticancer Therapy 10.3 (2010): 321329.

31. Lin Gigin and Yuen-Li Chung. "Current opportunities and challenges of magnetic resonance spectroscopy, positron emission tomography, and mass spectrometry imaging for mapping cancer metabolism In vivo". BioMed Research International (2014): 625095.

32. Wax Adam and Kevin J Chalut. "Nuclear morphology measurements with angle-resolved low coherence interferometry for application to cell biology and early cancer detection". Analytical Cellular Pathology 34.5 (2011): 207-222.

33. Kumar Saroj., et al. "Role of infrared spectroscopy and imaging in cancer diagnosis". Current Medicinal Chemistry 25.9 (2018): 1055-1072.

34. Rao Roopa S., et al. "Current aspects and future strategies in oral cancer research: A review". Journal of Medicine, Radiology, Pathology and Surgery 1.3 (2015): 8-13.

35. Pałasz Paulina., et al. "Contemporary diagnostic imaging of oral squamous cell carcinoma-a review of literature". Polish Journal of Radiology 82 (2017): 193.

36. Czerwonka Lukasz., et al. "High-resolution cone-beam computed tomography for assessment of bone invasion in oral cancer: Comparison with conventional computed tomography". Head and Neck 39.10 (2017): 2016-2020. 
37. Raja Jigna V and Vidya Priyadharshini. "Imaging In Oral Cancers-A Practical Approach". National Journal of Integrated Research in Medicine 7.1 (2016).

38. Mac Comb William S and Gilbert Hungerford Fletcher". Cancer of the Head and Neck. Williams and Wilkins (1967).

39. Gupta A., et al. "Spectroscopy in the Diagnosis of Oral Cancer". Journal of Dentofacial Sciences 4.1 (2015): 7-11.

40. Arya S., et al. "Imaging in oral cancer". Indian Journal of Radiology and Imaging 22 (2012): 195-208.

\section{Assets from publication with us}

- Prompt Acknowledgement after receiving the article

- Thorough Double blinded peer review

- Rapid Publication

- Issue of Publication Certificate

- High visibility of your Published work

Website: www.actascientific.com/

Submit Article: www.actascientific.com/submission.php

Email us: editor@actascientific.com

Contact us: +919182824667 